\title{
Application of BP neural network models and mind evolutionary algorithm in predicting stock composite indexes on Shanghai Stock Exchange
}

\author{
Guohao $\mathrm{Lu}^{1, \text { a }}$, Changping Xie ${ }^{2, \mathrm{~b}}$, Yingshu Zhang ${ }^{2, \mathrm{c}}$ and Shaomei Fang ${ }^{1, \mathrm{~d},{ }^{\star}}$ \\ ${ }^{1}$ College of Mathematics and Informatics, South China Agricultural University, Guangzhou 510642, \\ China \\ a460106895@qq.com, b229430694@qq.com, ‘469867110@qq.com, ddz90@scau.edu.cn \\ ${ }^{*}$ Corresponding author
}

\begin{abstract}
Keywords: Mind evolutionary algorithm, Back propagation neural network, Shanghai Stock Exchange Composite Index, Stock Prediction.
\end{abstract}

\begin{abstract}
Stock composite indexes prediction is an important issue in the financial world. A back propagation neural network (BPNN) with mind evolutionary algorithm (MEA) developed for the prediction of prices on Shanghai Stock Exchange is presented. The optimum weights and threshold values of BPNN are determined by MEA, which solves partial minimization of BPNN. Experiments are performed with Shanghai Stock Exchange stock to determine the effectiveness of the model. The results indicate that the accuracy rate of the proposed model is more than $70 \%$.
\end{abstract}

\section{Introduction}

With the development of the stock market, stock investment has become an important part of people's daily life, meanwhile, as an important branch of economic research, stock forecasting has always been a hot topic of financial prediction [1].

In the past two decades, a large body of research for predicting stock prices has been developed, such as decision trees, discriminant analysis and Markov model. Recently, back propagation neural networks (BPNN) have been proved to be useful research models to forecast stock prices [2]. However, there are still some drawbacks, when using BPNN, like the slow convergence rate and the low precision. To come over the shortcomings, some researchers have developed hybridized neural network techniques by combining optimization algorithm with BPNN [3]. Nowadays, a number of techniques including particle swarm optimization (PSO), ant colony algorithm (ACA) and genetic algorithm (GA) have been utilized to improve BPNN, the results of which showed the superiority of the neural networks.

In this paper, the back propagation neural network coupled with mind evolutionary algorithm (MEA) is proposed as a new method to predicting stock composite indexes on Shanghai Stock Exchange. Then the accuracy rate of more than $70 \%$ shows the effectiveness of the model.

\section{MEA-BPNN forecasting model}

Before training BPNN, we use MEA to optimize the initial weights and thresholds of BP neural network. First, according to BPNN topology, the solution space is mapped to the coding space and each solution of the problem corresponds each encoding. Because BPNN topology established in this paper is $8-10-1$, encoding length is $8 \times 10+10 \times 1+10+1=101$, we select reciprocal of mean square error of the training set as a score function of each individual and the population, and use MEA through iteration, output the best individual that as the best initial weights and thresholds, which is used to train BPNN. The flowchart of the MEA-BPNN algorithm is shown in Fig. 1. 


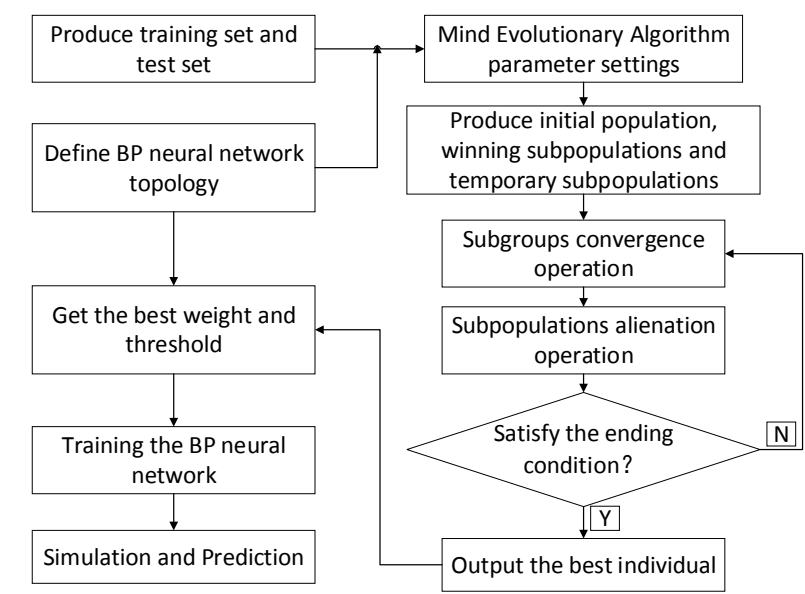

Fig. 1. MEA-BP Neural Network algorithm flowchart

\section{Model Building}

We use the optimized BP neural network to deal with past historical data of the Shanghai Stock Exchange Composite Index and to find the complex nonlinear relationship which could describe stock composite index direction, and then save it in the network, which help us forecast the future direction of index.

\subsection{The topological structure of BPNN}

\subsubsection{The input variable}

In this paper, we build eight inputs as the input variable including opening price, highest price, lowest price, closing price, trading volume, turnover price, change ratio and change amount of Shanghai Stock Exchange Composite Index in one day.

3.1.2 The output variable

We choose the next day's closing price of the Shanghai Exchange Composite Index as the output variable.

3.1.3 The number of hidden layer

According to the Kolmogorov theorem, we choose the BPNN with three-layer network (input layer, hidden layer and output layer) in the prediction.

3.1.4 The hidden layer nodes

Nowadays, most of researches define the hidden layer nodes according to the empirical formula and

trial-and-error method. The common experience formula is as follows

$$
m=\sqrt{n l}, m=\log _{2} n, m=\sqrt{n+l}+a,
$$

where $m$ represents the hidden layer nodes, $n$ represents the input layer nodes, $l$ represents the output layer nodes, $a$ is a constant between 1 to 10 [4]. Here, we make $a$ equal to 7, so the hidden layer nodes of this article is 10 .

3.1.5 The weights and thresholds

We determine the topological structure of BPNN and make the solution space mapped to the coding space of MEA. Then we can get the best initial weights and thresholds through the iteration of MEA.

3.1.6 The other parameters

(1) The number of iterations is 1000. (2) Error accuracy is 0.0001. (3) Learning rate is 0.05.

\subsection{The parameters of MEA}

The number of population, superior subpopulation, temporary subpopulation, subpopulation and iterations is $2000,5,5,20$ and 10 , respectively.

\subsection{Selecting and preprocessing of the data}

3.3.1 Selecting of the data 
The data in our study Netease financial market center (Information on http: //quotes.money.163. com/stock). After screening and eliminating invalid data, we ultimately select the daily transaction data of the Shanghai Stock Exchange Composite Index as the research data of model. They are the working days from April 21, 1995 to November 27, 2015. The trend of the closing price is shown in Fig. 2. We divide the data into the training data set, test set and validation set. The validation set include the transaction data of 10 days from November 16, 2015 to November 27, 2015. We randomly sort the transaction data of 4990 days from April 21, 1995 to November 15, 2015. We choose the transaction data of 4000 days in this 4990 days as the training set, and the remaining 990 days as the test set.

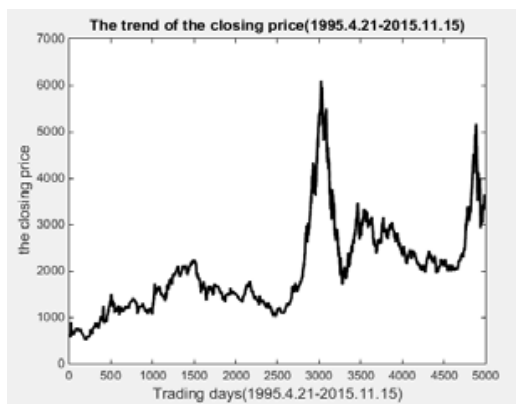

Fig. 2 The trend of the closing price

\subsubsection{Preprocessing of the data}

As the order of magnitude of the trading volume and trading turnover are greater than the others, it will result in a great error if we train the neural networks by original data. So both input variables and output variables are necessary in normalization processing.

Normalization processing is a common method of data processing before forecasting. In order to eliminate the difference in the orders of magnitude, we transform all the data into the number on $[0,1]$, We choose mapminmax () in MATLAB as the method of normalization, which is as below:

$$
x_{k}^{\prime}=\left(x_{k}-x_{\min }\right) /\left(x_{\max }-x_{\min }\right) \text {, }
$$

where $x_{k}^{\prime}$ represents the normalized data, $x_{k}$ represents the original input data, $x_{\min }$ represents the minimum number of data sequence, and $x_{\max }$ represents the maximum number of data sequence.

The normalized data not only eliminate the difference in the orders of magnitude but also substantially maintain the original characteristics of the data. As for the output data, we will make the anti-normalization process as following:

\subsection{Model evaluation criteria}

$$
x_{k}=x_{k}^{\prime}\left(x_{\max }-x_{\min }\right)+x_{\min }
$$

In order to analyze the prediction effect of this model, we choose the accuracy of trend as the evaluation index. The accuracy of trend is the ratio of the times that predicted and the expected trend is the same direction and the times of total forecast, which reflecting the forecasting odds ratio of model. At the same time, the accuracy of trend is generally demanded above $70 \%$.

\section{Interpretation of Result}

In this paper, the prediction model of Shanghai Stock Exchange Composite Index has been established. The simulation experiments are programed though MATLAB.

We randomly sort the transaction data of 4990 days from April 21, 1995 to November 15, 2015. We choose the transaction data of 4000 days in the 4990 days as the training set, and the remaining 990 days as the test set. First, we train MEA-BPNN through the train set. Then we use the successfully trained neural network to forecast the test set. At last, we forecast the latest data by this MEA-BPNN.

We have run 10 tests, and the accuracy of trend is as shown in Table 1. According to Table 1, we can see that the accuracy of trend is above $70 \%$ in each test. The average accuracy is $75.072 \%$. What's more, the highest accuracy is above $80 \%$, and the predicted results with high accuracy of the 
MEA-BPNN model are stable. According to Fig. 3, we can see that the predicted output and the expected output are fit basically. From Fig. 4 and Fig. 5, we can see that both the forecast error and the percentage almost hover around 0 . Table 2 is the comparison between predictive value and real value of the validation set which also shows the effective of the model.

Table 1 the accuracy of trend in 10 tests

\begin{tabular}{cccccc}
\hline The running order & 1 & 2 & 3 & 4 & 5 \\
\hline The accuracy of trend & 0.7446 & 0.8022 & 0.7554 & 0.7482 & 0.7626 \\
\hline The running order & 6 & 7 & 8 & 9 & 10 \\
\hline The accuracy of trend & 0.7338 & 0.7086 & 0.7626 & 0.7554 & 0.7338 \\
\hline
\end{tabular}

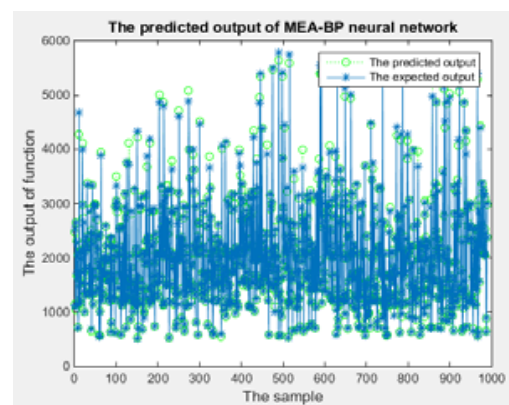

Fig. 3 The comparison

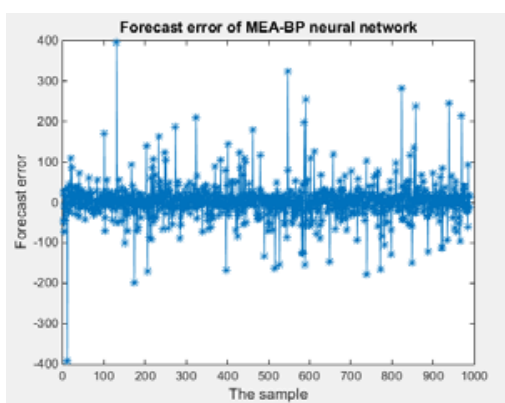

Fig. 4 Forecast error Fig. 5 The percentage of forecast error Table 2 The comparison between predictive value and real value of the validation set

\begin{tabular}{ccccc}
\hline Date & Real value & $\begin{array}{c}\text { Predictive } \\
\text { value }\end{array}$ & $\begin{array}{c}\text { The absolute } \\
\text { error }\end{array}$ & The relative error \\
\hline $2015 / 11 / 30$ & 3445.405 & 3448.001742 & 2.596741776 & 0.000753683 \\
$2015 / 11 / 27$ & 3436.303 & 3613.314815 & 177.0118146 & 0.051512284 \\
$2015 / 11 / 26$ & 3635.552 & 3630.439003 & -5.11299669 & -0.001406388 \\
$2015 / 11 / 25$ & 3647.93 & 3599.224299 & -48.70570146 & -0.0133516 \\
$2015 / 11 / 24$ & 3616.113 & 3595.475587 & -20.63741343 & -0.005707071 \\
\hline
\end{tabular}

\section{Conclusion}

In this paper, we use MEA to optimize BP neural network, which solves partial minimization of BP neural network and takes better forecast precision in the forecast of Shanghai Stock Exchange Composite Index. Meanwhile we prove the feasibility of BP neural network optimized by optimization algorithm. Then we built the MEA-BP neural network and made prediction on Shanghai Stock Exchange Composite Index. The accuracy of trend is above $70 \%$ in each test, which shows that the prediction model has a better predictive effect.

\section{Acknowledgements}

This work was financially supported by the national innovative training project for college students(201410564193), the Guangdong special funds for cultivation of technological innovation by university students (pdjh2015b0094), the Guangdong education innovation project for graduate students (2013SFKC03) and the NSFC (11271141).

\section{References}

[1] B. Shen, Stock Investment Analysis, Chongqing publishing house, Chongqing, 2002: 94.

[2] T. Kimoto, K. Asakawa, M. Yoda, etal, Stock Market Prediction System with Modular Neural Networks. Neural Networks, 1990 IJCNN, intermational Joint Conferenceon, 1990,Vol.1.1 6.

[3] W.X. Zhang, BP Neural Network Forecasting Model Based on PSO, Harbin Institute of Technology, Harbin, 2010.

[4]L.Q. Han, Artificial Neural Network Tutorial, Beijing University of Posts and Telecommunications publishing house, Beijing, 2006,pp. 74-75. 